\title{
Analysis of power generation and ice making system based on pressure and cold energy using of pipeline natural gas
}

\author{
Jingjing $X u^{1}$, Qifen $\mathrm{Li}^{1}$, Zongqin Hou${ }^{1}$, Guihai Jiao ${ }^{1}$, Lei Zhang ${ }^{1}$, Jialin Zhao', \\ Xiaoyu Liang ${ }^{1}$, Junpeng Mao ${ }^{1}$, Yuntao Zhu ${ }^{1}$ \\ ${ }^{1}$ College of energy and Mechanical Engineering, Shanghai University of Electric Power, China
}

\begin{abstract}
KEYWORD: Natural gas pipeline; Pressure and cold energy; Energy and exergy analysis
ABSTRACT: Natural gas transported in pipeline is at high pressures. But the pressure of natural gas is needed to be dropped to consumption valves before entering the user side. Available energy from the pressure reduction process of pipeline natural gas has been analyzed in this paper. A power generation and ice making system based on pressure and cold energy of the pipeline natural gas has been proposed to improve the operation economy of natural gas pipeline network. Finally, the energy and exergy analysis of the system is carried out. The results show that the system can significantly recycle the energy of the system.
\end{abstract}

\section{INTRODUCTION}

With the adjustment of national energy structure, the proportion of natural gas which is a kind of clean energy is increasing in primary energy consumption. The related data shows that the proportion will increase to more than $10 \%$ by 2020 . At the same time, Chinese natural gas pipeline network will develop rapidly, too.

Natural gas is always transported over long distance in high pressure pipeline. The pressure of the $3 \mathrm{rd}$ west-east natural gas pipeline in China is up to $12 \mathrm{MPa}$, which is much higher than consumption pressure. So natural gas in the transport process is needed to be compressed firstly to high pressure gas in the compressor station. But near to the user side, the pressure of natural gas is needed to be dropped to consumption valves, such as $1 \sim 3 \mathrm{MPa}$ or $0.2 \sim 0.8 \mathrm{MPa}$.

That is to say, in the process of natural gas transported by pipeline, there exists a great deal of high quality energy caused by the high mass flow rate and the high-pressure gas. In additional, the pressure drop process can bring considerable temperature drop in the natural gas, due to the Joule Thomson effect. However, the current regulator does not use this part of energy. So in the process of natural gas transportation, there exit a huge space of energy use and energy saving.

On the other hand, as the development of industrial and agricultural production, ice in the refrigerator car, cold storage, fishing, food industry and other departments have been widely used. The region of needing the ice is expanding. At present, the method of artificial ice can be divided into two kinds of method, indirect cooling and direct cooling. No matter what way we use to make ice, it needs to provide power for the refrigeration system.

Under the energy resources increasingly tense state of the union, pressure power and cold power recycled from the natural gas pipeline network can be used for electricity generation and ice-making, in a large extent, which will improve energy utilization rate and the economy of natural gas pipeline network operation. This paper focuses on the available energy analysis in the pressure reducing process of natural gas. And the energy and exergy analysis of power generation- ice making system based on the pipeline natural gas pressure and cold energy utilization.

\section{ANALYSIS OF AVAILABLE ENERGY FROM THE PRESSURE REDUCTION PROCESS OF PIPELINE NATURAL GAS}

Exergy is the maximum quantity of energy that can be converted to power in theory, which is called the available energy of the energy. The exergy analysis method is used to analyze the potential energy that can be used during the process of natural gas pressure in the pipe. 
Whether the process of natural gas pressure reduction is the throttling process or the isentropic process, from the perspective of thermodynamics, the system of natural gas pressure reduction is an open system.

Figure 1 shows that temperature entropy diagram of natural gas pressure reducing process can be considered as the isothermal transformation process and isobaric temperature process, that is to say the exergy of this process can be expressed as the temperature exergy caused by thermal unbalance under the condition of pressure unchanged and the pressure exergy caused by unbalanced force under the condition of a certain temperature and that is:

$e_{x, h}=e_{x, T}+e_{x, p}$

Where $e_{x, h}=$ Specific enthalpy exergy of natural gas, $\mathrm{kJ} / \mathrm{kg} ; e_{x, T}=$ Specific temperature exergy of natural gas, $\mathrm{kJ} / \mathrm{kg} ; e_{x, p}=$ Specific pressure exergy of natural gas.

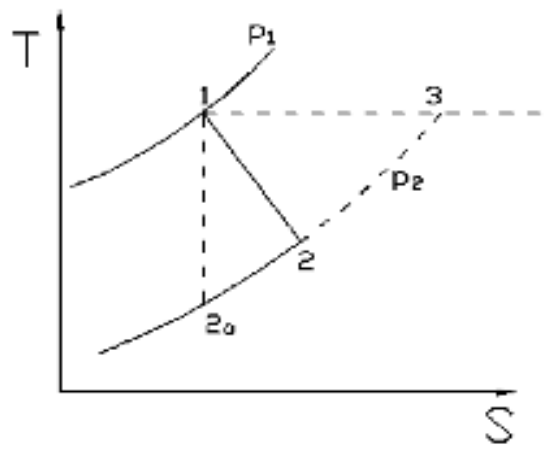

Figure 1. Temperature-entropy diagram of natural gas pressure reducing process.

The pressure exergy of natural gas is equivalent to the process of reducing the pressure of natural gas in the isothermal flow, that is:

$$
\begin{aligned}
e_{x, p} & =e_{x}\left(T_{0}, P_{1}\right)-e_{x}\left(T_{0}, P_{2}\right) \\
& =\int_{P_{2}}^{P_{1}} V d P=T_{0} R_{g} \ln \frac{P_{1}}{P_{2}}
\end{aligned}
$$

Where: $T_{0}=$ ambient temperature, $\mathrm{K} ; \mathrm{T}=$ Natural gas temperature after the change of state, $\mathrm{K} ; P_{1}$ $=$ Absolute pressure of natural gas before the change of state, $\mathrm{MPa} ; c_{p^{---}}$specific heat at constant pressure of Natural gas, $\mathrm{kJ} /(\mathrm{kg} \cdot \mathrm{K})$.

According to figure 1, pressure exergy and temperature exergy just shows that the quantity of the available energy natural during the process of gas pressure reducing, in the actual process of decompression, due to the different pressure reduction equipment, such as throttle valve, turbine expansion machine, the pressure energy that was used are also different. Take pressure reduction station for example, of which the average inlet pressure, the average mass flow rate and the average temperature are respectively taken $5.0 \mathrm{MPa}, 37304.8 \mathrm{~kg} / \mathrm{h}$ and $15^{\circ} \mathrm{C}$. Natural gas has been considered as pure methane. With the turbo expander taken as pressure equipment, by Aspen simulation machine, Shaft power and the outlet temperature of natural gas are calculated under the different distribution pressure, in addition ,the pressure exergy and cold exergy are also calculated according to the formula (2), and (3). Draw the pressure exergy, cold exergy, and shaft power calculation results into its trend diagram, as shown in figure 2. 


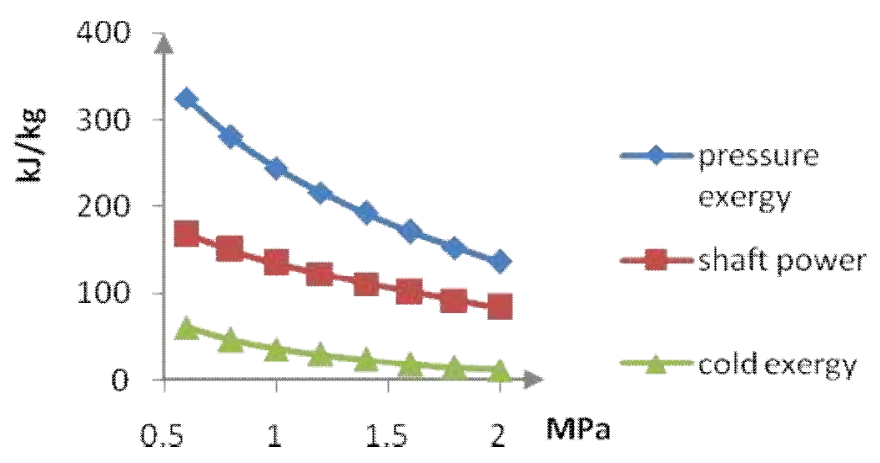

Figure 2. The available energy of natural gas pipeline pressure.

We can see from Figure 2:1) the pressure exergy calculated according to the formula (2) is not equal to the shaft power of expansion equipment; 2) the trend of pressure exergy is the same as that of the shaft power during the process of expansion : In the case of constant inlet pressure, the two increase with the decrease of the outlet pressure; 3 )The changing trend of the cold exergy shows that after the expansion the capacity of the natural gas absorption heat is enhanced with the decrease of the outlet pressure.

\section{POWER GENERATION AND ICE MAKING SYSTEM BASED ON PRESSURE AND COLD ENERGY OF PIPELINE NATURAL GAS}

There are pressure energy and cold energy which can be used as the energy source in the process of natural gas pressure reduction. The pressure energy can be used to generate electricity, the cold energy can be used to make ice, so The electric energy is obtained while the gas pressure is reduced, The ice is gained without additional energy consumption, while the temperature of natural gas increasing. The system flow chart is shown in figure 3.

From figure 3, natural gas in high pressure flow into the turbo expander firstly and the pressure energy is used to drive a generator, while gas temperature has a considerable drop after expansion. Gas in low temperature go into salt pond passing cold energy to ice water by salt water, and then the cold energy of the low temperature natural gas is further utilized in the reservoir. At last the electric heater is used to ensure the quality of the gas to downstream users.

To sum up, the system can be used to recover the pressure and cold energy of natural gas by turbo expander and the equipment of ice making system, which will improve the energy utilization efficiency of the pipe network.

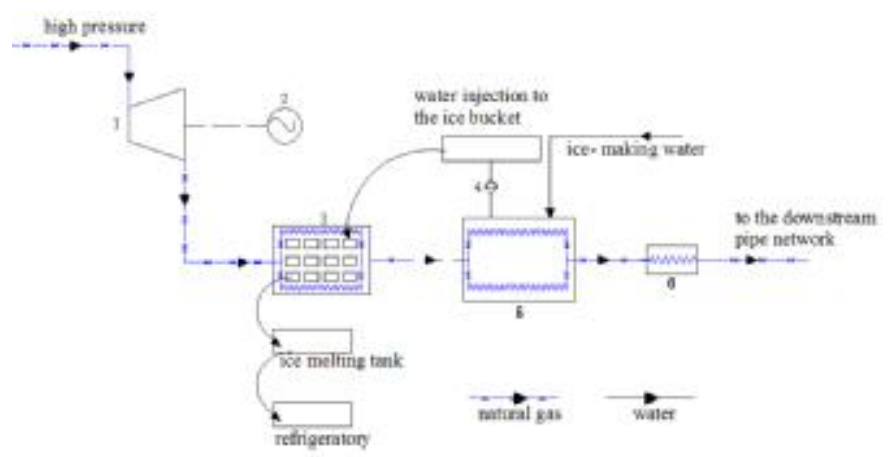

1-Turbo expander; 2- Electric generator;3- Brine pool;4-Pump; 5- Reservoir;6- Electric heater

Figure 3. The flow chart of power generation-ice making system.

The part of pressure energy recycled - part of power generation 
High pressure gas into the Turbo expander and using the pressure energy drive a generator, So that the pressure energy of the gas pressure reducing process can be recovered and converted into electrical energy

\section{The part of cold energy recycled - part of ice making}

After decompression the gas temperature has a great range of fall, the cold gas at low temperature can make ice. Low temperature gas go into salt pond passing cold energy to ice water by salt water, then the ice is got. After salt water heating, the temperature of natural gas is in $-10{ }^{\circ} \mathrm{C}$ or so, then we can use this part of cold energy the for pre-cooling the ice water, in this way the initial water temperature into the ice bucket was reduced, thereby reducing the cold energy the salt water pool need to produce the same ice, which can increase the amount of ice in the same conditions. In a word, The system recovered cold energy of natural gas after pressure reduction by using the salt -ice making method.

\section{ANALYSIS OF POWER GENERATION AND ICE MAKING SYSTEM BASED ON PRESSURE AND COLD ENERGY OF PIPELINE NATURAL GAS}

The model used in this study is considered salt pond and reservoir as heat exchangers. In this open system conservation of energy at control volume is steady state. Besides, the changes in potential energy and kinetic are assumed to be negligible.

The pressure drop of heat exchanger was assumed to be negligible. Entropy efficiency of turbo expander is $80 \%$.

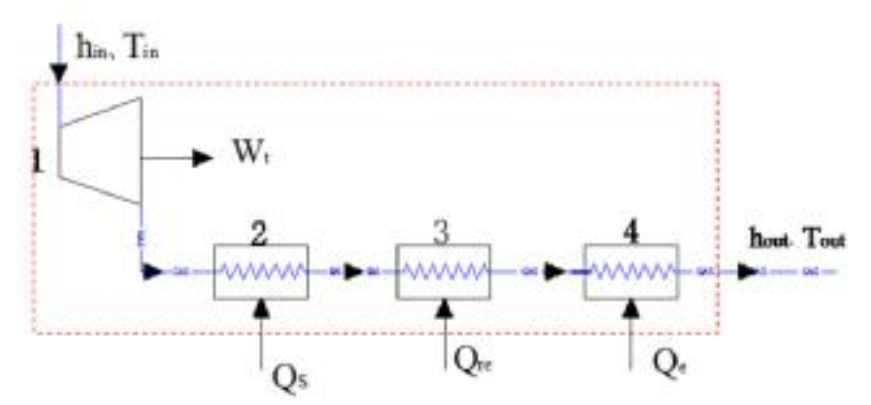

1-Turbo expander; 2- Brine pool;3- Reservoir;4- Electric heater

Figure 4. The inlet and outlet energies into/from the control volume.

Energy analysis of the system

Take the control volume as figure 4 . In this system, there exist the output work, and cold energy. $Q_{s}$ is the heat natural gas absorption in brine pool, $Q_{r e}$ is the heat natural gas absorption in the storage pool, $Q_{e}$ is the heat natural gas absorption from the electric heater, $Q_{0}$ is the heat exchange between the system and the environment. The energy balance equation of this stable flow opening system can be written as:

$$
H_{\text {gas }, \text { in }}-H_{\text {gas }, \text { out }}=\mathrm{W}_{i}-Q_{0}-Q_{s}-Q_{r e}-Q_{e}
$$

Where: $W_{i=} W_{t}$, and $W_{t}$ is the shaft power of Turbo expander; $H_{\text {gas, in }}$ and $H_{\text {gas,out }}$ are respectively the natural gas enthalpy inlet and outlet the control volume.

In this system, according to the practical significance of the system, the energy efficiency of the control volume could be defined as : 
$\eta_{1}=\frac{Q_{s}+Q_{r e}+\mathrm{W}_{t}}{H_{\mathrm{gas}, \text { in }}-H_{\text {gas }, \text { out }}+Q_{e}}$

Exergy analysis of the system

According to the exergy balance equation of the stable flow system:

$E_{x, Q}+\sum_{n} \Delta E_{x, H_{n}}=\mathrm{W}_{t}+i$

Where: $E_{x, Q}$ is the heat exergy between the heat source and the environment; And $W_{t}$ is the shaft power of Turbo expander, and without considering the bearing friction, internal power is equal to the shaft power; $\Delta E_{x, H}$ is the difference between inlet and outlet natural gas enthalpy exergy of the control volume; $i$ is exergy loss of the system.

In this system, there exist the output work, and cold exergy. Assuming that is the heat exchange between the system and the environment. The black box model was shown in figure 5 .

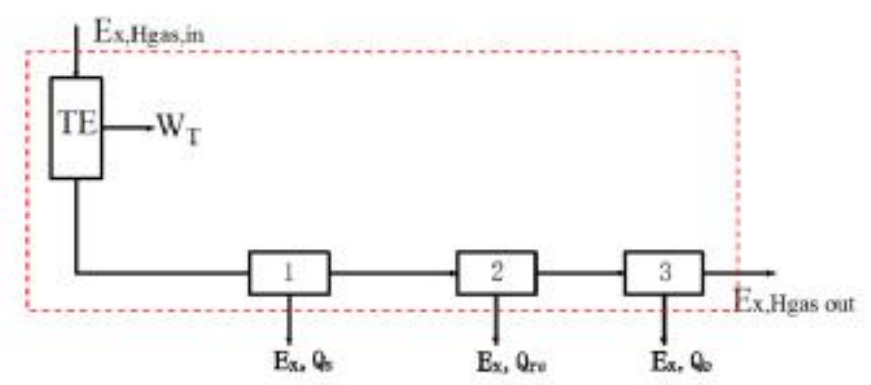

1- Electric generator;2- Brine pool;3-Pump;

Figure 5. The inlet and outlet exergies into/from the control volume.

The exergy balance equation of the system is written as:

$E_{x, H_{\text {gas }, \text { in }}}-E_{x, H_{\text {gas out }}}=\mathrm{W}_{t}+i+E_{x, Q_{s}}+E_{x, Q_{\text {re }}}+E_{x, Q_{e}}$

Where: $W_{t}$ is the shaft power of Turbo expander; $E_{x, Q}, E_{x, r e}$ and $E_{x, e}$ are respectively natural gas exergy in the brine pool, the storage pool and the heat exchanger. $E_{x, \text { Hgas, in }}$ and $E_{x, \text { Hgas,out }}$ are the enthalpy exergy of natural gas. And $E_{x, H}=q_{m} \times\left(h-T_{0} s\right)$.

The energy efficiency of the control volume could be defined as:

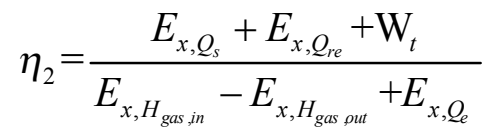

\section{Results of the system energy and exergy analysis}

On the basis of the second part, outlet pressure of expander is taken $2.0 \mathrm{MPa}$, and the mass flow natural gas is taken $37304.8 \mathrm{~kg} / \mathrm{h}$. Assuming the water temperature at the inlet of the brine pool is $15^{\circ} \mathrm{C}$, and the water temperature at the outlet of reservoir is $0^{\circ} \mathrm{C}$, and the natural gas is transported to the downstream user at $0^{\circ} \mathrm{C}$. Combined with the Aspen simulation results of the system, the calculation can be carried out according to the formula (6) and (7):

$\eta_{1}=\frac{Q_{s}+Q_{r e}+\mathrm{W}_{t}}{H_{\text {gas }, \text { in }}-H_{\text {gas }, \text { out }}+Q_{e}}=1.56$ 
$\eta_{2}=\frac{E_{x, Q_{s}}+E_{x, Q_{\text {re }}}+\mathrm{W}_{t}}{E_{x, H_{\text {gas }, \text { in }}}-E_{x, H_{\text {gas out }}}+E_{x, Q_{e}}}=76.9 \%$

\section{CONCLUSION}

Through the above analysis, it can be seen that the greater the pressure difference between the pressure drop stations is, the more the pressure energy and the cold energy can be used. What's more, power generation-ice making system based on pressure and cold energy of pipeline natural gas, can not only avoid energy loss during the operation of natural gas pipeline, which effectively improved the energy utilization efficiency, but also can gain additional electric energy and ice. Due to the utilization of the cold energy in the system, the energy utilization efficiency of the system is $1.56>1$ 。 The exergy efficiency of the system is only $76.9 \%$, which shows that the system can be further optimized.

\section{ACKNOWLEDGEMENTS}

This work was financially supported by the Research Project of Shanghai Science and Technology Committee (13dz1201700).

\section{REFERENCES}

[1] Clifford Howard. \& Patrick Oosthuizen. \& Brant Peppley. 2011. An investigation of the performance of a hybrid turboexpander-fuel cell system for power recovery at natural gas pressure reduction stations. Applied Thermal Engineering $31: 2165-2170$.

[2] Wang Qiang. \& Li Yanzhong. \& Wang Jiang. 2004. Analysis of power cycle based on cold energy of liquefied natural gas and low-grade heat source. Applied Thermal Engineering 24 :539-548.

[3] Li xiaxi. \& Jin Yazhou. \& Gao Min. \& Wang Suo. \& Han Lixin. \& Yan Lin. \& Xu Wendong. \& Yuan Dan. 2004. Study of the pressure energy generation potential and application prospect of Beijing natural gas pipeline network. Gas Technology 10: 10-15.

[4] A.Arabkoohsar. \& M.Farzaneh-Gord. \& M. DeymiDashtebayaz. \& L.Machado. \& R.N.N. Koury. 2015. A new design for natural gas pressure reduction points by employing a turbo expander and a solar heating set. Renewable Energy 81: 235-250.

[5] Mehmet Alparslan Neseli. \& Onder Ozgener. \& Leyla Ozgener. 2015. Energy and exergy analysis of electricity generation from natural gaspressure reducing stations. Energy Conversion and Management93: 109-120. 